\title{
Calculation of magnetic properties of metals by means of the magnetic-field-containing relativistic tight-binding approximation method
}

\author{
Masahiko Higuchi \\ Department of Physics, Faculty of Science, Shinshu University, Matsumoto 390-8621, Japan \\ Dipendra Bahadur Hamal \\ Department of Natural Sciences (Physics), Kathmandu University, Dhulikhel, Kavre 6250, Nepal
}

Katsuhiko Higuchi

Graduate School of Advanced Sciences of Matter, Hiroshima University, Higashi-Hiroshima 739-8527, Japan

(Received 20 May 2016; revised manuscript received 8 August 2016; published 24 May 2017)

\begin{abstract}
Magnetic properties of metals are investigated through electronic structure calculations based on the recently proposed magnetic-field-containing relativistic tight-binding approximation (MFRTB) method [Phys. Rev. B 91, 075122 (2015)]. It is found that electronic energy bands for a metal immersed in a uniform magnetic field have a cluster structure in which multiple energy bands lie within a small energy width. Each cluster corresponds to an energy level that is derived on the basis of the semiclassical approximation. While the cluster is responsible for the de Haas-van Alphen (dHvA) oscillations, constituent energy bands of the cluster cause additional oscillation peaks of the magnetization that are not explained by the conventional Lifshitz-Kosevich formula. Also, the energy width of the cluster leads to the reduction of the amplitude of the dHvA oscillations, which can be observed as the pseudo Dingle temperature and/or the overestimation of the curvature of the Fermi surface.
\end{abstract}

DOI: 10.1103/PhysRevB.95.195153

\section{INTRODUCTION}

Measurements of de Haas-van Alphen (dHvA) oscillations in metals [1-4] are widely used in investigating the shape of the Fermi surface, cyclotron effective mass, and relaxation time for scattering of electrons [5-7]. In order to describe the dHvA oscillations, we need electronic states of metals immersed in a uniform magnetic field. For this purpose, there are conventionally two kinds of methods. One is based on the effective Hamiltonian that is obtained by replacing the rest mass of electrons with the effective mass in the Hamiltonian for a free electron immersed in a uniform magnetic field [5,8,9]. The effects of the periodic potential are taken into account via the effective mass. Although quantized energy levels (so-called Landau levels) are obtained within this approximation, we cannot explain the dependence of the dHvA oscillations on the direction of the magnetic field [9]. This is due to an oversimplified argument such that the characteristics of individual metals are taken into consideration only through the effective mass [9].

Another method to describe electronic states of metals immersed in a uniform magnetic field is based on the semiclassical approximation [4,5,8,9]. Hereafter we will call this method the "semiclassical approach". In the semiclassical approach, instead of directly solving the Schrödinger or Dirac equation, both the Bohr-Sommerfeld quantization rule that is obtained within the semiclassical approximation and the equation of motion for a Bloch electron in the magnetic field are used in order to get quantized energy levels (semiclassical energy levels) $[4,5,8,9]$. This method leads to the usual description for the dHvA oscillations such that every time one semiclassical energy level crosses the Fermi energy with increasing the magnetic field, one oscillation of the magnetization is produced [5,8,9]. The Lifshitz-Kosevich (LK) formula [10] is derived by means of semiclassical energy levels, and is commonly employed in analyzing the dHvA oscillations [5-10]. On the basis of the LK formula, one can evaluate the extremal cross-sectional area of the Fermi surface normal to the magnetic field from the oscillation period [5-10]. Also, according to the LK formula, the temperature and magnetic field dependences of the oscillation amplitude give information on the cyclotron effective mass and relaxation time for scattering of electrons, respectively [5-10].

Recently, we developed the magnetic-field-containing relativistic tight-binding approximation method (MFRTB method) that enables us to directly solve the Dirac equation for crystalline materials immersed in a uniform magnetic field $[11,12]$. This method is a first-principles calculation method that is applicable to various kinds of realistic materials immersed in a uniform magnetic field [11]. In the previous work [11], we applied this method to crystalline silicon immersed in the magnetic field as the first step toward revealing the mechanism of the elastic softening and its suppression observed in boron-doped silicon [13-16]. It is shown that the energy band structures have an explicit dependence on the magnetic field, and that a recursive energy spectrum, which is similar to the Hofstadter butterfly diagram [17], is observed. Through this application, the MFRTB method is illustrated to be useful for revealing the electronic structure of materials immersed in a uniform magnetic field [11].

Following the above-mentioned application, the MFRTB method is also used to describe the dHvA oscillations [12]. It is shown that the dHvA oscillations can be reproduced by the MFRTB method [12]. We also found that the oscillation period of the conventional LK formula is a good approximation to that of the MFRTB method in experimentally available magnetic fields, while in high magnetic fields it deviates from the period of the MFRTB method [12].

In this paper, we present a detailed description of the magnetic oscillation phenomena by means of the MFRTB 
method. Especially, we investigate the following nonconventional magnetic oscillation phenomena that cannot be explained by the semiclassical approach:

(1) additional oscillation peaks of the magnetization beyond the conventional dHvA oscillations, and

(2) an unexpected reduction of the oscillation amplitude.

Specifically, we show that additional oscillation peaks are caused by the characteristic energy band structure of metals immersed in the magnetic field. It is also revealed from the analysis of the oscillation amplitude that the oscillation amplitude is unexpectedly reduced in the high magnetic field region, where "unexpectedly" means that the reduction of the oscillation amplitude cannot be explained by the conventional LK formula. As shown latter, this reduction would lead to the observation of the "pseudo" Dingle temperature [18] and/or overestimation of the curvature of the Fermi surface, even though the relaxation time of electron scattering is very long.

The organization of this paper is as follows. The MFRTB method is applied to a simple cubic lattice system with $s$ electrons. In Sec. II, we explain the reason why the MFRTB method is applied to such a simple system. In Sec. III, a detailed description of the dHvA oscillations is presented by using the electronic structure calculated by the MFTRB method. In Sec. IV, the appearance, origin, and observability of additional oscillation peaks are discussed on the basis of a detailed investigation of the electronic structure calculated by the MFRTB method. In Sec. V, we discuss the limit of the semiclassical approach through the estimation of the cyclotron effective mass. The results of the analysis of the oscillation amplitude are shown in Sec. VI. Finally, concluding remarks are presented in Sec. VII.

\section{APPLICATION OF THE MFRTB METHOD TO A SIMPLE CUBIC LATTICE SYSTEM}

The MFRTB method is applied to a simple cubic lattice system with $s$ electrons that is immersed in a uniform magnetic field [12]. In this section, we explain the reason why we apply the MFRTB method to a hypothetical simple cubic lattice with $s$ electrons instead of real materials.

As mentioned in Sec. I, we intend to investigate nonconventional oscillation phenomena that cannot be explained by the semiclassical approach. For this purpose, rigorous results of the semiclassical approach, such as semiclassical energy levels and the period and amplitude of the magnetic oscillation, are indispensable. This is because, by investigating the discrepancy between such rigorous results of the semiclassical approach and the corresponding results of the MFRTB method, we can discuss the origin of the nonconventional oscillation phenomena.

In order to obtain such rigorous results of the semiclassical approach, we need rigorous $\boldsymbol{E}$ - $\boldsymbol{k}$ curves for the zero magnetic field case. This is because rigorous results of the semiclassical approach are obtained from the extremal cross section of the Fermi surface, cyclotron effective mass, and curvature of the Fermi surface, and these are calculated by using the $E$ - $\boldsymbol{k}$ curves for the zero magnetic field case. For example, if the cyclotron effective mass was not rigorously calculated, then we could not obtain semiclassical energy levels rigorously, which causes a difficulty in associating the energy levels obtained by the
MFRTB method with the semiclassical ones. Accordingly, it would be difficult to discuss the origin of nonconventional oscillation phenomena if the cyclotron effective mass was not rigorously calculated. In this work, as the model system such that the $E$ - $\boldsymbol{k}$ curves for the zero magnetic field case can be obtained rigorously, we adopt a simple cubic lattice system with $s$ electrons. This enables us to accurately discuss the origin of the nonconventional oscillation phenomena that cannot be explained by the semiclassical approach.

Thus, we apply the MFRTB method to the simple cubic lattice with $s$ electrons immersed in the magnetic field. In calculations that will be shown later, we use the same relativistic TB parameters as those used in the previous works $[11,12]$. Also, the magnitude of the magnetic field is assumed to be given by

$$
B=\frac{h}{e a^{2}} \frac{p}{q},
$$

where $p$ and $q$ are relatively prime integers, and $a$ denotes the lattice constant $[11,12,17]$.

\section{DESCRIPTION OF THE de HAAS-van ALPHEN OSCILLATIONS THROUGH THE MFTRB METHOD}

In this section, we explain how the dHvA oscillations are described on the basis of the electronic structure that is calculated by the MFRTB method. Figure 1 shows the magnetic field dependences of the total energy and magnetization. The horizontal axis of Fig. 1 is $(p / q)^{-1}$, which is inversely propositional to $B$ [Eq. (1)]. Oscillations of the total energy and magnetization are clearly seen in Fig. 1. In order to describe the oscillatory behavior, we calculate densities of states (DOSs) for several magnetic fields that are indicated by (2a)-(2d) in Fig. 1. Resultant DOSs for the magnetic fields (2a)-(2d) are shown in Figs. 2(a)-2(d), respectively. Peak positions of the DOS can be classified into two types: one is that peak positions increase with $p / q$, and the other is that

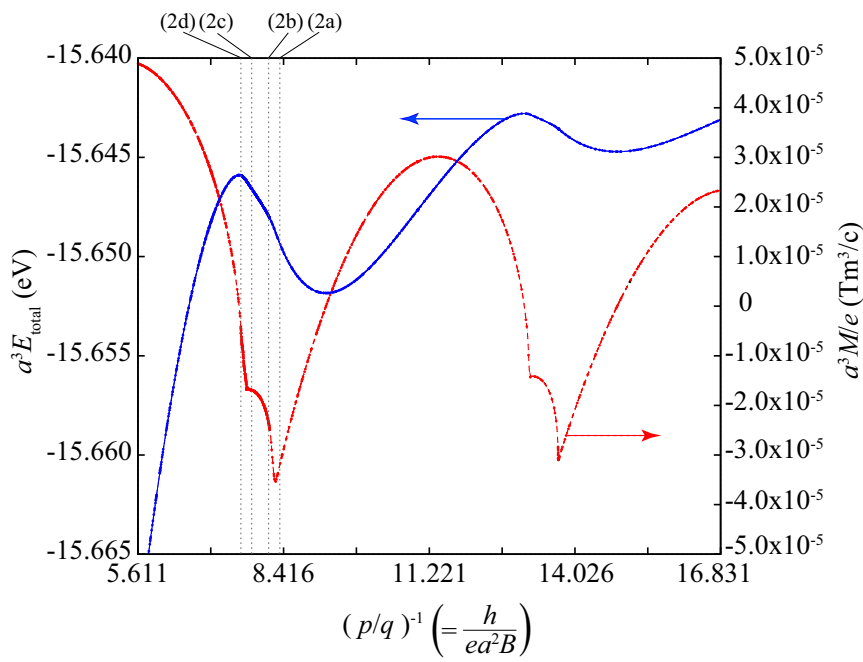

FIG. 1. Dependences of the total energy (blue) and magnetization (red) on the inverse of the magnitude of the magnetic field in the range $p / q=0.0594-0.178$. Symbols (2a), (2b), (2c), and (2d) indicate the magnetic fields at which we calculate the DOSs [see Figs. 2(a)-2(d)]. 


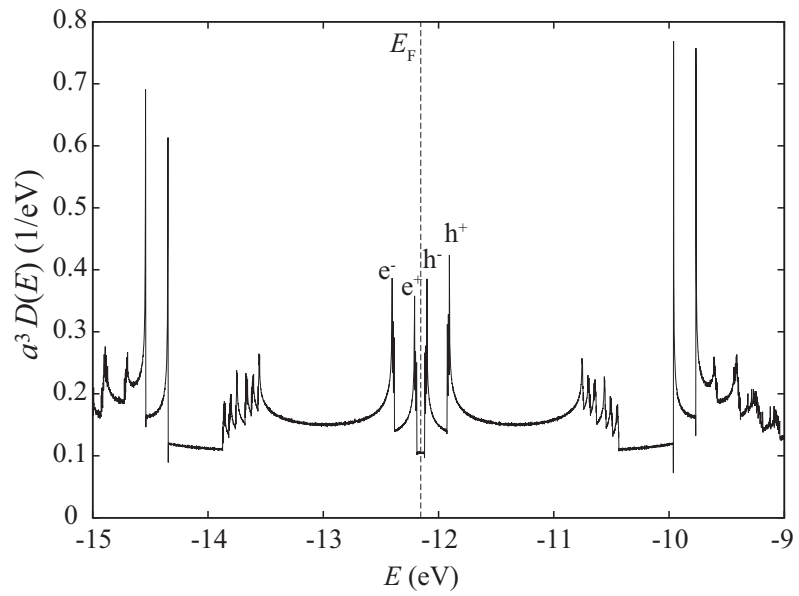

(a) $p / q=20 / 167$

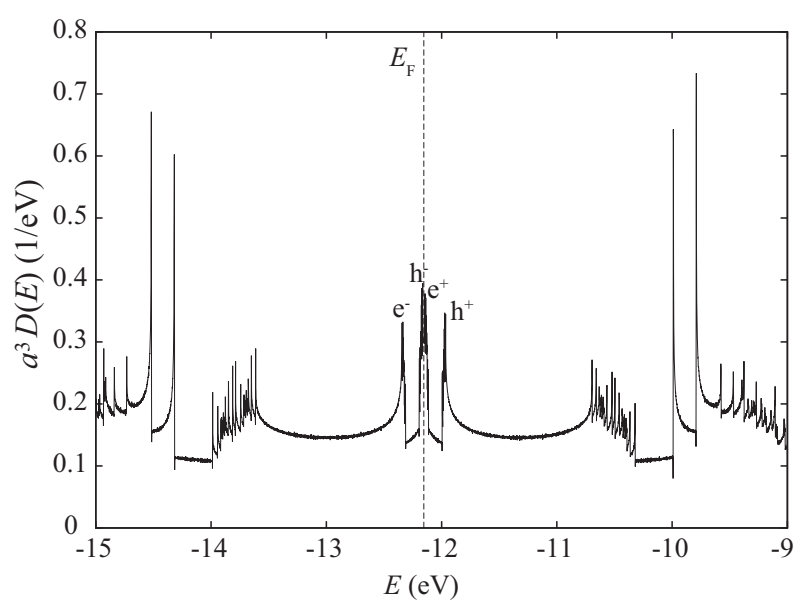

(b) $p / q=20 / 163$

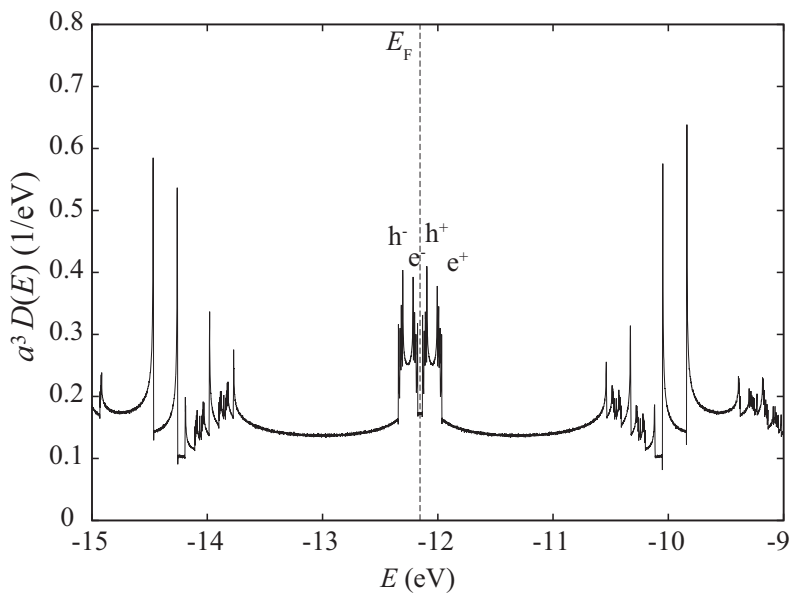

(c) $p / q=21 / 163$

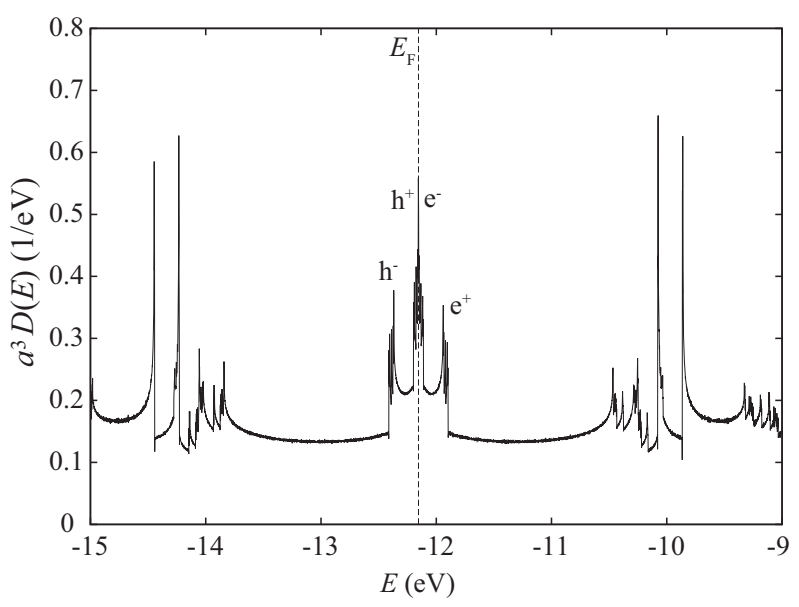

(d) $p / q=22 / 167$

FIG. 2. Magnetic field dependence of the DOSs for systems immersed in high magnetic fields. (a) DOS for the system immersed in the magnetic fields (2a) of Fig. 1. (b) DOS for the system immersed in the magnetic fields (2b) of Fig. 1. (c) DOS for the system immersed in the magnetic fields (2c) of Fig. 1. (d) DOS for the system immersed in the magnetic fields (2d) of Fig. 1.

they decrease with $p / q$. In Figs. 2(a)-2(d), peak positions of the former type are denoted by $\mathrm{e}^{+}$and $\mathrm{e}^{-}$, and those of the latter type are denoted by $\mathrm{h}^{+}$and $\mathrm{h}^{-}$, respectively. The pair of peaks $\left(\mathrm{e}^{+}, \mathrm{e}^{-}\right)$or $\left(\mathrm{h}^{+}, \mathrm{h}^{-}\right)$corresponds to the Zeeman splitting of spin states. The existence of two types of energy levels can be explained also by the semiclassical approach. Namely, there exist electron and hole orbitals on the constant energy surface in $\boldsymbol{k}$ space for the case of a simple cubic lattice system with $s$ electrons. For the present system, the cyclotron effective mass for the electron orbital is positive, while that for the hole orbital is negative. Since the interval between two energy levels is inversely proportional to the cyclotron effective mass according to the semiclassical approach [8], semiclassical energy levels that come from electron (or hole) orbitals increase (or decrease) with the magnetic field. Thus, we can associate $\mathrm{e}^{+}$and $\mathrm{e}^{-}\left(\mathrm{h}^{+}\right.$and $\left.\mathrm{h}^{-}\right)$with the semiclassical energy levels for electron (hole) orbitals.

Next, we explain the relation between magnetic oscillations (Fig. 1) and DOSs [Figs. 2(a)-2(d)]. It is expected that the magnetic field dependence of occupied energy levels near the Fermi energy has a major effect on that of the total energy. In the case of the magnetic fields (2a), the highest and next highest occupied energy states are $\mathrm{e}^{+}$and $\mathrm{e}^{-}$, respectively [Fig. 2(a)]. Therefore, the total energy is expected to increase with $p / q$ because their peak positions increase with $p / q$. Indeed, the total energy increases with $p / q$ as shown in Fig. 1 . With the increase of the magnetic field from (2a) to (2b), the highest occupied energy states switch from $\mathrm{e}^{+}$to $\mathrm{h}^{-}$. In this situation, the highest occupied energy states $\left(\mathrm{h}^{-}\right)$decrease with $p / q$, while the next highest occupied energy states $\left(\mathrm{e}^{-}\right)$oppositely increase with $p / q$. The effect of this switch is expected to appear in the slope of the total energy. As shown in Fig. 1, the slope of the total energy changes between (2a) and (2b). This implies that not only the magnetic field dependence of the highest occupied energy levels but also that of the next highest occupied energy levels have a major effect on that of the total energy. Corresponding to the change of the slope of the total energy, the magnetization exhibits the characteristic peak between ( $2 a)$ and (2b) as seen in Fig. 1. When the magnetic field increases from (2b) to (2c), the highest occupied energy states switch from $\mathrm{h}^{-}$to $\mathrm{e}^{-}$. Correspondingly, the slope of the total energy slightly decreases between (2b) and (2c), as 


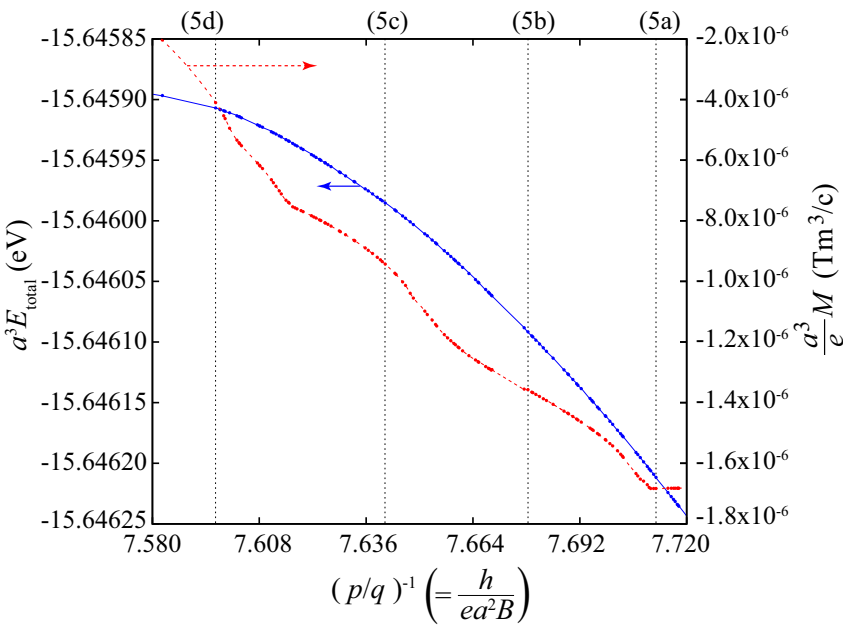

FIG. 3. Dependences of the total energy (blue) and magnetization (red) on the inverse of the magnitude of the magnetic field in the range $p / q=0.130-0.132$. Symbols (5a) $-(5 \mathrm{~d})$ indicate the magnetic fields at which we calculate the DOSs [see Figs. 5(a)-5(d)].

shown in Fig. 1. Further increase of the magnetic field [(2c) $\rightarrow(2 \mathrm{~d})]$ leads to a switch of the highest occupied energy states from $\mathrm{e}^{-}$to $\mathrm{h}^{+}$. This switch results in a change of the slope of the total energy, which causes the kink in the magnetization between (2c) and (2d) (Fig. 1). At the magnetic field (2d) both the highest and next highest occupied energy levels decrease with $p / q$, so that the total energy decreases with $p / q$ (Fig. 1).

Thus, every time the energy levels that correspond to the semiclassical energy level pass over the Fermi energy, a magnetic oscillation is produced. Namely, the dHvA oscillations are produced by the repeated crossing of semiclassical energy levels to the Fermi energy. This description of the magnetic oscillation is similar to that by the semiclassical approach [8].

\section{ADDITIONAL OSCILLATION PEAKS}

In this section, it is shown that additional oscillation peaks, which cannot be explained by the LK formula, are observed in the magnetic field dependence of the magnetization. Also, the origin and observability of additional oscillation peaks are discussed.

\section{A. Additional oscillation peak and its origin}

Figure 3 shows the magnified view of Fig. 1. It is seen in Fig. 3 that there exist rugged peaks (additional oscillation peaks) in the magnetization together with the oscillations that can be explained by the LK formula. Of course, additional oscillation peaks cannot be explained by the LK formula.

In order to clarify the origin of additional oscillation peaks, we calculate energy band structures for four magnetic fields that are indicated by (5a)-(5d) in Fig. 3. Figure 4 shows the energy band structure for the case of magnetic field (5d), which corresponds to the DOS of Fig. 2(d). The horizontal axis of Fig. 4 denotes the special $\boldsymbol{k}$ points in the magnetic first Brillouin zone of the simple cubic lattice [12]. In Fig. 4, energy bands become nearly flat between the $Z$ point $(0,0, \pi / a)$ and $R$ point $(\pi / a, 0, \pi / a)$ as well as between the $M$ point

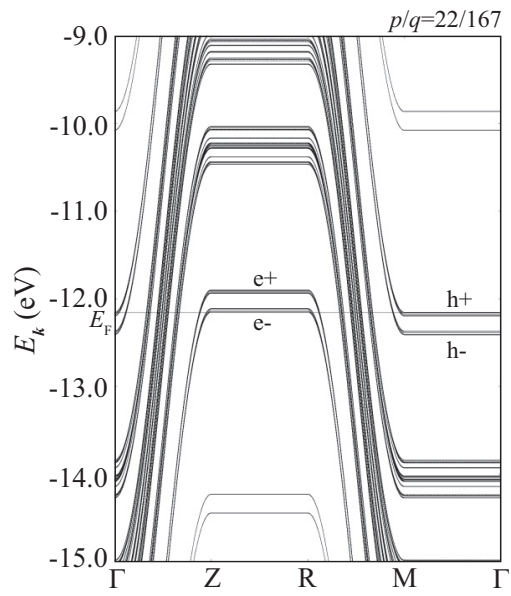

FIG. 4. Energy band structure for the case of the magnetic field (5d) that is indicated in Fig. 3. This energy band structure corresponds to the DOS of Fig. 2(d). Symbols $Z, R, M$, and $\Gamma$ in the horizontal axis denote special $\boldsymbol{k}$ points in the magnetic first Brillouin zone [12]. Coordinates of special $\boldsymbol{k}$ points $Z, R, M$, and $\Gamma$ are given by $(0,0, \pi / a),(\pi / a, 0, \pi / a),(\pi / a, \pi / q a, 0)$, and $(0,0,0)$, respectively.

$(\pi / a, \pi / a q, 0)$ and $\Gamma$ point $(0,0,0)$. There exist two nearly flat bands around the Fermi energy between the $Z$ point and $R$ point. These two bands correspond to $\mathrm{e}^{-}$and $\mathrm{e}^{+}$of Fig. 2(d) because it is confirmed that their energy levels increase with $p / q$. On the other hand, two nearly flat bands around the Fermi energy between the $M$ point and $\Gamma$ point correspond to $\mathrm{h}^{-}$and $\mathrm{h}^{+}$of Fig. 2(d). It seems from Fig. 4 that $\mathrm{e}^{-}, \mathrm{e}^{+}, \mathrm{h}^{-}$, and $\mathrm{h}^{+}$ consist of a lot of nearly flat bands.

In order to clarify effects of these nearly flat bands on the magnetic oscillation, energy bands around the Fermi energy are shown in Figs. 5(a)-5(d) for the cases of magnetic fields (5a)-(5d). Note that Fig. 5(d) is the magnified view of Fig. 4. A lot of nearly flat bands can be seen in Fig. 5(d) more clearly than in Fig. 4. In Fig. 5(d), the cluster [19] of nearly flat bands above the Fermi energy corresponds to the DOS peak $\mathrm{e}^{-}$of Fig. 2(d), while the cluster of nearly flat bands below the Fermi energy corresponds to the DOS peak $\mathrm{h}^{+}$of Fig. 2(d). Thus, the cluster of nearly flat bands lying within a small energy width corresponds to the semiclassical energy level. This "fine energy-level structure" plays a crucial role in appearance of the additional oscillation peaks that will be mentioned below.

Comparing Fig. 3 with Figs. 5(a)-5(d), we can see that additional oscillation peaks are produced when energy bands that are constituents of the cluster cross the Fermi energy. Roughly speaking, there exist three blocks of energy bands in the cluster along both $Z-R$ and $M-\Gamma$ lines [Figs. 5(a)-5(d)]. This feature of the fine energy-level structure is maintained during the change of the $p / q$ ratio from $92 / 709$ to $22 / 167$ as shown in Figs. 5(a)-5(d). When the first block crosses the Fermi energy with the increase of the magnetic field from (5a) to $(5 \mathrm{~b})$, the slope of the total energy is expected to change, similarly to the case of the semiclassical energy level (Sec. III). Indeed, the kink of the magnetization appears between (5a) and (5b) as shown in Fig. 3. When the second block crosses to the Fermi energy, the magnetization has a depressed shape between (5b) and (5c). This shape is also due to the change 

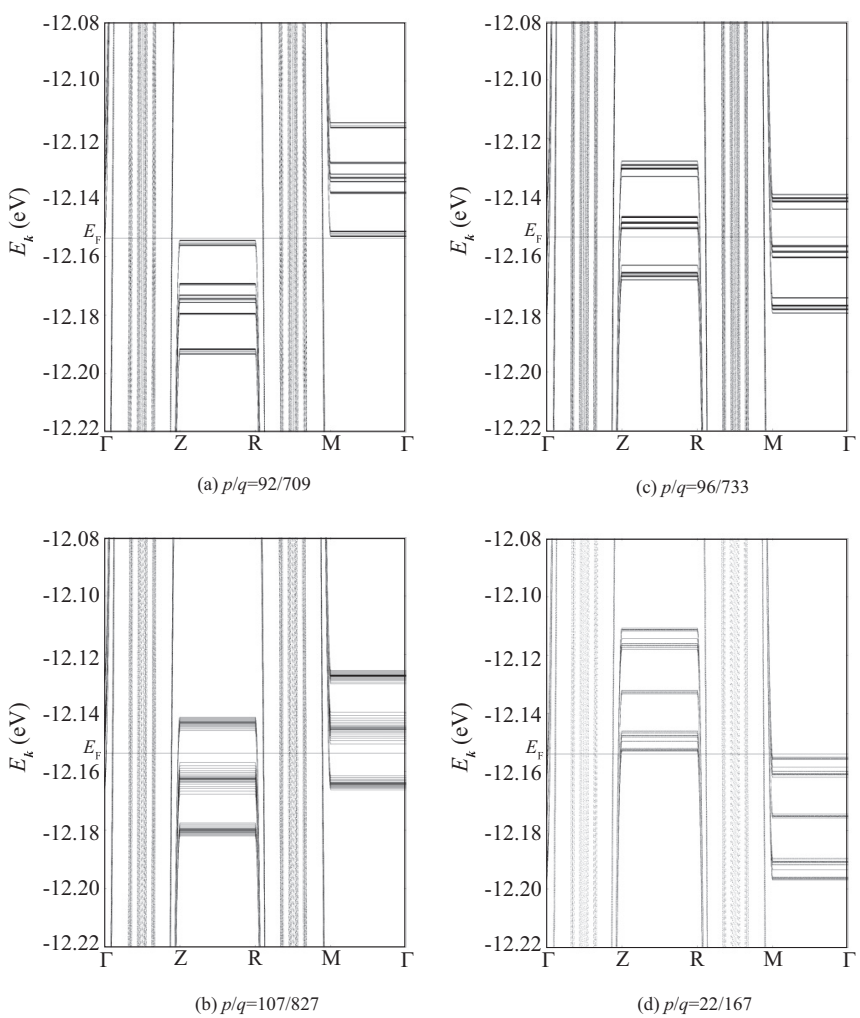

FIG. 5. Magnetic field dependence of the energy band structure for the system immersed in a magnetic field. (a) In the case of the magnetic field (5a) of Fig. 3. (b) In the case of the magnetic field (5b) of Fig. 3. (c) In the case of the magnetic field (5c) of Fig. 3. (d) In the case of the magnetic field (5d) of Fig. 3.

of the slope of the total energy. Similarly to the cases of the first and second blocks, the crossing of the third block to the Fermi energy leads to a depressed shape of the magnetization between (5c) and (5d). Thus, additional oscillation peaks originate from the fine energy-level structure that is revealed by the MFRTB method. It is should be noted that the crossing of only one energy band may cause the additional oscillation peak. If we take the steps of the magnetic field more finely in the horizontal axis of Fig. 3, then more rugged peaks will appear in the magnetization.

\section{B. Relation between the fine energy-level structure and magnetic oscillations}

In this subsection, the relation between the fine energy-level structure and magnetic oscillations is investigated in more detail. For this aim, we will review the energy band structure obtained by the MFRTB method [11,12]. In the MFRTB method, the magnitude of the magnetic field is given by Eq. (1). As discussed in Ref. [11], the energy band structure strongly depends on the value of the rational number $p / q$. Let us consider energy band structures for two magnetic fields $B \propto 1 / q^{\prime}$ and $B \propto p / q$, the magnitudes of which are nearly equal to each other, i.e., $1 / q^{\prime} \approx p / q$. We have $2 q^{\prime}$ energy bands in the case of $B \propto 1 / q^{\prime}$, while in the case of $B \propto p / q$ we have $2 q\left(\approx 2 p q^{\prime}\right)$ energy bands that are nearly $p$ times more than that in the case of $B \propto 1 / q^{\prime}$. This is understood by the fact that the period of the translation symmetry along the $y$ direction in the case of $B \propto p / q$ is nearly $p$ times longer than that in the case of $B \propto 1 / q^{\prime}$ due to the relation $q^{\prime} p \approx q[11,12]$. Namely, due to the folding of the magnetic first Brillouin zone, $p$ energy gaps may be induced at the boundaries of the magnetic first Brillouin zone in the case of $B \propto p / q$. Therefore, each energy band in $B \propto 1 / q^{\prime}$ splits into $p$ energy bands, so that $2 q\left(\approx 2 q^{\prime} p\right)$ energy bands appears in the case of $B \propto p / q$ [11].

Since the energy bands in general overlap each other, it is expected that the number of allowed bands is approximately proportional to that of energy bands $\left(2 q^{\prime}\right)$ in the case of $B \propto 1 / q^{\prime}$, which has been directly confirmed through numerical calculations [11]. Namely, one allowed band consists of several energy bands. Let us consider again two magnetic fields cases: $B \propto 1 / q^{\prime}$ and $B \propto p / q$ with $p / q \approx 1 / q^{\prime}$. Since the individual energy band in the case of $B \propto 1 / q^{\prime}$ splits into $p$ energy bands in the case of $B \propto p / q$ [11], an allowed band in the case of $B \propto 1 / q^{\prime}$ would split into multiple allowed bands in the case of $B \propto p / q$; the number of multiple allowed bands would be proportional to $p$. In the previous paper [11], we refer such multiple allowed bands as "cluster". If an allowed band in the case of $B \propto 1 / q^{\prime}$ consists of $w$ energy bands, then the corresponding cluster in the case of $B \propto p / q$ consists of more energy bands, the number of which would be proportional to $w p$.

We take the case of $p / q=22 / 167$ as an example. As mentioned in Sec. III, energy levels that are denoted as $\mathrm{e}^{+}$, $\mathrm{e}^{-}, \mathrm{h}^{+}$, and $\mathrm{h}^{-}$in Fig. 2(d) correspond to the semiclassical energy levels. These energy levels correspond to nearly flat bands in the energy band structure as shown in Fig. 4. It is confirmed from Fig. 5(d) that the nearly flat bands of $\mathrm{e}^{-}$ consist of $22(=p)$ energy bands. The same is true for $\mathrm{e}^{+}, \mathrm{h}^{+}$, and $\mathrm{h}^{-}$. This means that the semiclassical energy levels $\left(\mathrm{e}^{+}\right.$, $\mathrm{e}^{-}, \mathrm{h}^{+}$, and $\mathrm{h}^{-}$) correspond to the above-mentioned cluster that contains $p$ energy bands. Thus, it is revealed by the MFRTB method that the semiclassical energy level splits into multiple energy bands that form a cluster.

At the end of this subsection, we comment on the difference between the conventional dHvA oscillations and additional oscillation peaks on the basis of the above-mentioned knowledge about the energy band structure. The constituent energy bands of the cluster have the same magnetic field dependence as each other. Therefore, the global dependence of the total energy on the magnetic field (conventional dHvA oscillations) is determined by the magnetic field dependence of the cluster. The crossing of constituent energy bands of the cluster to the Fermi energy has a small but definite influence on the magnetic field dependence of the total energy, which appears as the additional oscillation peaks of the magnetization. Consequently, we can say that the additional oscillation peaks come from the energy bands that form the cluster while the conventional dHvA oscillations are produced by the clusters that correspond to semiclassical energy levels.

\section{Observability of additional oscillation peaks}

In this subsection, we discuss the observability of additional oscillation peaks. As mentioned in the previous section, additional oscillation peaks originate from energy bands that 
are constituents of the cluster. Since the energy width of the cluster (energy band width) increases with $p / q[11,20]$, the splitting of energy bands in the cluster would increase with $p / q$. It is therefore expected that the observation of additional oscillation peaks becomes more feasible as $p / q$ increases. Inversely, as $p / q$ decreases, we need to control the value of $p / q$ (the magnitude of the magnetic field) with good accuracy in order to observe additional oscillation peaks. Therefore, there is a possibility of observing additional oscillation peaks if we measure the magnetic field dependence of the magnetization in the high magnetic field region (high $p / q$ region) with a sufficiently fine step of the magnetic field.

There is another case of observing additional oscillation peaks. As shown in Fig. 3, additional oscillation peaks appear around the magnetic field (5c) $(p / q=96 / 733)$. In the present calculations we take $0.543 \mathrm{~nm}$ as $a$, which is equal to the lattice constant of crystalline silicon, so that $p / q=96 / 733$ corresponds to $B=1837 \mathrm{~T}$ due to Eq. (1). If we consider the system, the period of which is longer than $a=0.543 \mathrm{~nm}$, then the magnitude of the magnetic field becomes smaller for $p / q=96 / 733$. For example, if we consider the superlattice system with the period $10 a$, then $p / q=96 / 733$ corresponds to $B=18.37 \mathrm{~T}$, which is the experimentally available magnetic field.

More specifically, graphene placed on hexagonal boron nitride (hBN) [21-24] seems to be one of the suitable systems for observing additional oscillation peaks. This is because the slight difference in the lattice constant between the graphene and hBN leads to the moiré superlattice potential [21-24], the period of which is as long as about $15(\mathrm{~nm})$. Indeed, the characteristic gap structure of the energy spectrum, which reflects the fine energy level structure and is referred to as the Hofstadter butterfly diagram, is experimentally observed in this system [21-24]. Therefore, it is expected that additional oscillation peaks would be observed in a system with a long period such as graphene placed on a hBN substrate.

\section{CYCLOTRON EFFECTIVE MASS}

As mentioned in the previous section, the cluster that corresponds to the semiclassical energy level has an energy bandwidth. It is expected that the energy bandwidth may have an effect on the amplitude of the dHvA oscillations. This is because it is known that the broadening of the energy level leads to a reduction of the oscillation amplitude [18]. According to the conventional LK formula, the amplitude of the dHvA oscillations depends on the cyclotron effective mass, curvature of the Fermi surface, and relaxation time for scattering of electrons [10]. Since the MFRTB method can deal with only zero-temperature systems, it is difficult to simultaneously analyze contributions of three quantities to the oscillation amplitude. Therefore, before discussing the effect of the energy band width of the cluster on the oscillation amplitude (Sec. VI), in this section, we separately estimate the cyclotron effective mass alone through the MFRTB method.

First, we explain how to estimate the cyclotron effective mass. For this purpose, let us start by reviewing the semiclassical approach for a Bloch electron in a magnetic field [8]. In the semiclassical approach, the cyclotron effective mass is defined by

$$
m_{c}\left(E, k_{z}\right)=\frac{\hbar^{2}}{2 \pi} \frac{d A\left(E, k_{z}\right)}{d E},
$$

where $A\left(E, k_{z}\right)$ is the cross-sectional area of the constant energy surface in a plane normal to the magnetic field. According to the semiclassical approach, the electron goes around the edge of the cross section with the frequency $e B / 2 \pi m_{c}\left(E, k_{z}\right)$. The quantized energy levels in the semiclassical approach satisfy the Bohr-Sommerfeld quantization rule and Bohr's correspondence principle [8]. According to Bohr's correspondence principle, the difference between two adjacent energy levels is given by Planck's constant times the frequency of classical motion at the energy levels [8]. Therefore, if the quantized energy level is denoted as $\varepsilon_{v}\left(k_{z}\right)$, then Bohr's correspondence principle is expressed by

$$
\text { (A) } \varepsilon_{v+1}\left(k_{z}\right)-\varepsilon_{v}\left(k_{z}\right)=\frac{\hbar e B}{m_{c}\left(\varepsilon_{v}\left(k_{z}\right), k_{z}\right)} \text {. }
$$

It should be noted that Eq. (3) holds approximately for energy levels with very high quantum number $v$ [8]. When we consider energy levels with very high quantum numbers $v$, $\varepsilon_{v+1}\left(k_{z}\right)-\varepsilon_{v}\left(k_{z}\right)$ is expected to be much less than $\varepsilon_{v+1}\left(k_{z}\right)$ and $\varepsilon_{v}\left(k_{z}\right)$. In this case, it is expected that both $m_{c}\left(\varepsilon_{v+1}\left(k_{z}\right), k_{z}\right)$ and $m_{c}\left(\left\{\varepsilon_{v+1}\left(k_{z}\right)+\varepsilon_{v}\left(k_{z}\right)\right\} / 2, k_{z}\right)$ are close to $m_{c}\left(\varepsilon_{v}\left(k_{z}\right), k_{z}\right)$ because the difference $\varepsilon_{v+1}\left(k_{z}\right)-\varepsilon_{v}\left(k_{z}\right)$ is much less than $\varepsilon_{v+1}\left(k_{z}\right)$ and $\varepsilon_{v}\left(k_{z}\right)$. Therefore, we can rewrite Eq. (3) as

$$
\begin{gathered}
\text { (B) } \varepsilon_{v+1}\left(k_{z}\right)-\varepsilon_{v}\left(k_{z}\right)=\frac{\hbar e B}{m_{c}\left(\varepsilon_{v+1}\left(k_{z}\right), k_{z}\right)}, \\
\text { (C) } \varepsilon_{v+1}\left(k_{z}\right)-\varepsilon_{v}\left(k_{z}\right)=\frac{\hbar e B}{m_{c}\left(\left\{\varepsilon_{v+1}\left(k_{z}\right)+\varepsilon_{v}\left(k_{z}\right)\right\} / 2, k_{z}\right)} .
\end{gathered}
$$

The DOS obtained by the semiclassical approach has a sharp peak when the energy is identical to $\varepsilon_{v}\left(k_{z}^{\text {ext }}\right)$, where $k_{z}^{\text {ext }}$ denotes the wave number such that $A\left(E, k_{z}\right)$ has a extremal value, i.e., $\left(\partial A\left(E, k_{z}\right) / \partial k_{z}\right)_{k_{z}=k_{z}^{\text {ext }}}=0$ [8]. This means that interval of peak positions of the DOS corresponds to $\hbar e B / m_{c}\left(\varepsilon_{v}\left(k_{z}^{\mathrm{ext}}\right), k_{z}^{\mathrm{ext}}\right)$, $\hbar e B / m_{c}\left(\varepsilon_{v+1}\left(k_{z}^{\mathrm{ext}}\right), k_{z}^{\mathrm{ext}}\right)$ or $\hbar e B / m_{c}\left(\left\{\varepsilon_{v+1}\left(k_{z}^{\text {ext }}\right)+\varepsilon_{v}\left(k_{z}^{\text {ext }}\right)\right\} / 2, k_{z}^{\text {ext }}\right)$ depending on the choice of the expression of Bohr's correspondence principle [(A), (B), or (C)]. Therefore, we may reasonably identify the interval of peak positions of the DOS that is calculated by the MFRTB method with $\hbar e B / m_{c}\left(\varepsilon_{v}\left(k_{z}^{\mathrm{ext}}\right), k_{z}^{\mathrm{ext}}\right)$, $\hbar e B / m_{c}\left(\varepsilon_{v+1}\left(k_{z}^{\mathrm{ext}}\right), k_{z}^{\mathrm{ext}}\right)$ or $\hbar e B / m_{c}\left(\left\{\varepsilon_{v+1}\left(k_{z}^{\text {ext }}\right)+\varepsilon_{v}\left(k_{z}^{\text {ext }}\right)\right\} / 2, k_{z}^{\text {ext }}\right)$.

Using Eqs. (3)-(5), we estimate three kinds of the cyclotron effective mass from intervals of peak positions of the DOS that are calculated by the MFRTB method. Hereafter, we denote three kinds of cyclotron effective mass by $m_{c}^{\operatorname{MFRTB}(\mathrm{A})}\left(E, k_{z}^{\mathrm{ext}}\right)$, $m_{c}^{\mathrm{MFRTB}(\mathrm{B})}\left(E, k_{z}^{\text {ext }}\right)$, and $m_{c}^{\mathrm{MFRTB}(\mathrm{C})}\left(E, k_{z}^{\text {ext }}\right)$, corresponding to Eqs. (3)-(5). It should be noted that if the quantum number is sufficiently high that Bohr's correspondence principle holds with good accuracy, then $m_{c}^{\operatorname{MFRTB}(\mathrm{A})}\left(E, k_{z}^{\text {ext }}\right)$, $m_{c}^{\mathrm{MFRTB}(\mathrm{B})}\left(E, k_{z}^{\mathrm{ext}}\right)$, and $m_{c}^{\mathrm{MFRTB}(\mathrm{C})}\left(E, k_{z}^{\mathrm{ext}}\right)$ will be approximately equal to each other. Inversely, differences between the three kinds of cyclotron effective masses indicate the inaccuracy of the semiclassical approach.

In the case of the simple cubic lattice, $A\left(E, k_{z}\right)$ has extremal values at $k_{z}^{\mathrm{ext}}=\pi / a$ and 0 for the electron and 


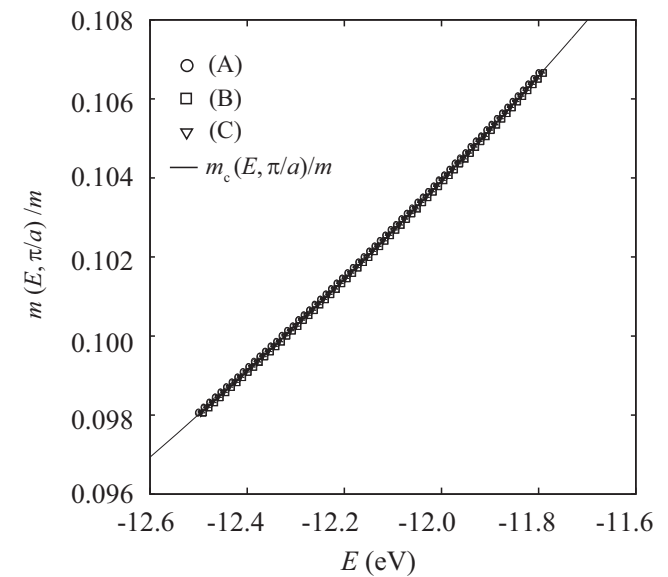

FIG. 6. Energy dependences of $m_{c}^{\operatorname{MFRTB}(\mathrm{A})}\left(E, k_{z}^{\mathrm{ext}}\right) / m$, $m_{c}^{\operatorname{MFRTB}(\mathrm{B})}\left(E, k_{z}^{\text {ext }}\right) / m, \quad m_{c}^{\operatorname{MFRTB}(\mathrm{C})}\left(E, k_{z}^{\text {ext }}\right) / m \quad$ and $\quad m_{c}\left(E, k_{z}^{\text {ext }}\right) / m$ for the electron orbital $\left(k_{z}^{\mathrm{ext}}=\pi / a\right)$ in the case of $p / q=1 / 1427 \approx 6.998 \times 10^{-4}(B=9.82 \mathrm{~T})$.

hole orbitals, respectively. Figure 6 shows energy-dependences of $m_{c}^{\mathrm{MFRTB}(\mathrm{X})}\left(E, k_{z}^{\text {ext }}\right) / m(\mathrm{X}=\mathrm{A}, \mathrm{B}$, and $\mathrm{C})$ of the electron orbital $\left(k_{z}^{\text {ext }}=\pi / a\right)$ in the case of $p / q=1 / 1427 \approx 6.998 \times$ $10^{-4}(B=9.82 \mathrm{~T})$. For comparison, the rigorous value of $m_{c}\left(E, k_{z}^{\text {ext }}\right) / m$ that is calculated from Eq. (2) is also shown in Fig. 6 by the solid line. It is found that the three kinds of the cyclotron effective masses $m_{c}^{\mathrm{MFRTB}(\mathrm{X})}\left(E, k_{z}^{\mathrm{ext}}\right) / m(\mathrm{X}=\mathrm{A}$, $\mathrm{B}$, and $\mathrm{C})$ are in a good agreement with $m_{c}\left(E, k_{z}^{\text {ext }}\right) / m$. Also, $m_{c}^{\operatorname{MFRTB}(\mathrm{X})}\left(E, k_{z}^{\mathrm{ext}}\right) / m(\mathrm{X}=\mathrm{A}, \mathrm{B}$, and $\mathrm{C})$ are approximately equal to each other. The differences between $m_{c}\left(E, k_{z}^{\text {ext }}\right) / m$ and $m_{c}^{\mathrm{MFRTB}(\mathrm{X})}\left(E, k_{z}^{\text {ext }}\right) / m(\mathrm{X}=\mathrm{A}, \mathrm{B}$, and $\mathrm{C})$ are about $-0.06 \%, 0.06 \%$, and $-0.0002 \%$, respectively. These agreements mean that Bohr's correspondence principle holds with good accuracy in the case of $p / q=1 / 1427$.

Figures 7(a) and 7(b) show the magnetic field dependences of $m_{c}^{\operatorname{MFRTB}(\mathrm{X})}\left(E_{F}, k_{z}^{\text {ext }}\right)$ for the electron orbital $\left(k_{z}^{\text {ext }}=\pi / a\right)$ and hole orbital $\left(k_{z}^{\text {ext }}=0\right)$, respectively. Vertical axes of Figs. 7(a) and 7(b) denote the difference between $m_{c}\left(E_{F}, k_{z}^{\text {ext }}\right)$ and $m_{c}^{\operatorname{MFRTB}(\mathrm{X})}\left(E_{F}, k_{z}^{\text {ext }}\right)\left(k_{z}^{\text {ext }}=\pi / a\right.$ and 0$)$, which is given by

$$
\begin{aligned}
& \Delta m_{c}^{\operatorname{MFRTB}(\mathrm{X})}\left(E_{F}, k_{z}^{\mathrm{ext}}\right) \\
& =\frac{m_{c}^{\operatorname{MFRTB}(\mathrm{X})}\left(E_{F}, k_{z}^{\mathrm{ext}}\right)-m_{c}\left(E_{F}, k_{z}^{\mathrm{ext}}\right)}{m_{c}\left(E_{F}, k_{z}^{\mathrm{ext}}\right)} .
\end{aligned}
$$

It is found from Figs. 7(a) and 7(b) that absolute values of $\Delta m_{c}^{\operatorname{MFRTB}(\mathrm{X})}\left(E_{F}, k_{z}^{\mathrm{ext}}\right)\left(k_{z}^{\mathrm{ext}}=\pi / a\right.$ and 0$)$ increase with the magnetic field for the three cases $(\mathrm{X}=\mathrm{A}, \mathrm{B}, \mathrm{C})$. Also, it is confirmed from Figs. 7(a) and 7(b) that differences between three kinds of cyclotron effective masses $\left[m_{c}^{\operatorname{MFRTB}(\mathrm{X})}\left(E_{F}, k_{z}^{\text {ext }}\right)\right.$ $(\mathrm{X}=\mathrm{A}, \mathrm{B}$, and $\mathrm{C})$ ] also increase with the magnetic field. These tendencies suggest that the accuracy of semiclassical energy levels gets worse with increasing magnetic field. This can be understood by considering the maximum quantum number. Namely, Bohr's correspondence principle is valid for energy levels with very high quantum number [8]. The maximum quantum number is roughly estimated by the ratio of $E_{F}$ and $\hbar e B / m_{c}\left(E_{F}, k_{z}^{\text {ext }}\right)$. This ratio becomes of the order of $10^{3}$ in the case of $B \sim 10 \mathrm{~T}\left(p / q \sim 7 \times 10^{-4}\right)$, while it is

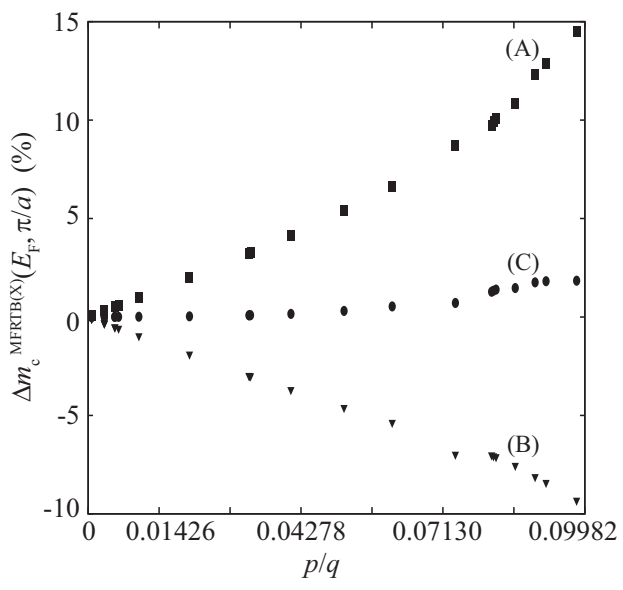

(a) $k_{\mathrm{z}}^{\mathrm{ext}}=\pi / a$

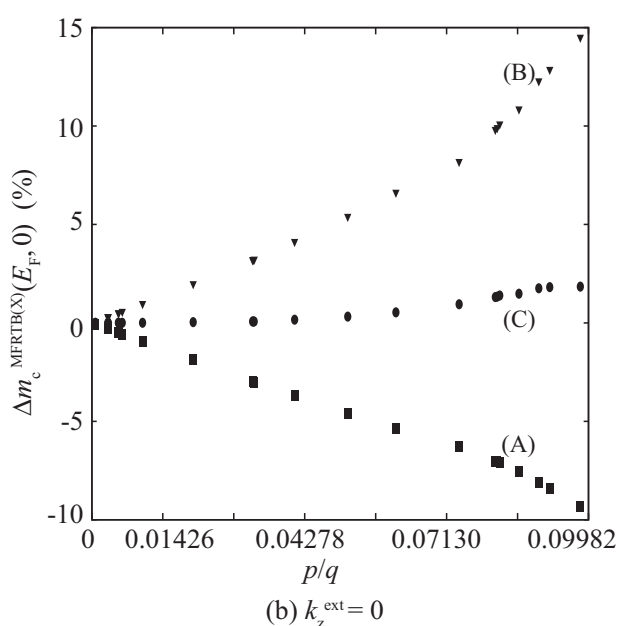

FIG. 7. Magnetic field dependences of $\Delta m_{c}^{\mathrm{MFRTB}(\mathrm{X})}\left(E_{F}, k_{z}^{\mathrm{ext}}\right)$ $(\mathrm{X}=\mathrm{A}, \mathrm{B}$, and $\mathrm{C})$ for (a) the electron orbital $\left(k_{z}^{\mathrm{ext}}=\pi / a\right)$ and (b) the hole orbital $\left(k_{z}^{\text {ext }}=0\right)$.

about 10 in the case of $B \sim 10^{3} \mathrm{~T}\left(p / q \sim 7 \times 10^{-2}\right)$. Thus, the maximum quantum number increases with $B$, so that the accuracy of semiclassical energy levels gradually gets worse in the high magnetic field.

It should be noted that the absolute value of $\Delta m_{c}^{\operatorname{MFRTB}(\mathrm{C})}\left(E_{F}, k_{z}^{\text {ext }}\right)$ is smaller than those of $\Delta m_{c}^{\operatorname{MFRTB}(\mathrm{A})}\left(E_{F}, k_{z}^{\text {ext }}\right)$ and $\Delta m_{c}^{\operatorname{MFRTB}(\mathrm{B})}\left(E_{F}, k_{z}^{\text {ext }}\right)$. Therefore, we had better use Eq. (5) instead of Eqs. (3) and (4) in order to estimate the cyclotron effective mass from the DOS that is obtained by the MFRTB method or by experiments such as photoelectron spectroscopy.

\section{ANALYSIS OF THE AMPLITUDE OF THE dHvA OSCILLATIONS}

The amplitude of the dHvA oscillations is usually analyzed on the basis of the LK formula that includes the effect of the scattering of electrons [5-7]. The effect of the scattering of electrons is incorporated into the LK formula by treating the quantized energy level as the broadened energy level with the width $\hbar / \tau$, where $\tau$ is a relaxation time [18]. This broadening leads to a reduction of the oscillation amplitude [18]. In the present MFRTB method, the scattering of electrons is not taken 
TABLE I. Resultant values of parameters for low $p / q$ regions.

\begin{tabular}{lcccr}
\hline \hline Range of $p / q$ & Period $\left(10^{-4} / \mathrm{T}\right)$ & $\gamma$ & $A^{\prime \prime}\left(E_{F}, k_{z}^{\text {ext }}\right)$ & $T_{\mathrm{D}}(\mathrm{K})$ \\
\hline $6.972 \times 10^{-4}-7.070 \times 10^{-4}$ & 3.85826 & 0.500 & 8.6253430 & $5.38 \times 10^{-7}$ \\
$3.079 \times 10^{-3}-3.270 \times 10^{-3}$ & 3.85855 & 0.495 & 8.6260636 & $7.61 \times 10^{-7}$ \\
$5.069 \times 10^{-3}-5.666 \times 10^{-3}$ & 3.85827 & 0.499 & 8.6260636 & $8.63 \times 10^{-7}$ \\
\hline \hline
\end{tabular}

into consideration. However, the cluster that corresponds to the semiclassical energy level appears to have an energy width as mentioned in Sec. IV. Therefore, it is expected that the energy width of the cluster will cause the reduction of the oscillation amplitude even though the scattering of electrons is not taken into consideration. In this section, the oscillation amplitude is analyzed through the MFRTB method.

\section{A. Analysis method}

The LK formula for the total energy density at $0 \mathrm{~K}$ is given by [10]

$$
\begin{aligned}
& E_{\text {total }}=\sqrt{\frac{e^{5}}{8 \pi^{7} \hbar}} \sum_{l=1} \sum_{k_{z}^{\text {ext }}} \frac{\cos \left(\pi l \frac{g m_{c}\left(E_{F}, k_{z}^{\text {ext }}\right)}{2 m}\right) R_{D} B^{5 / 2}}{l^{5 / 2} m_{c}\left(E_{F}, k_{z}^{\text {ext }}\right) \sqrt{\left|A^{\prime \prime}\left(E_{F}, k_{z}^{\mathrm{ext}}\right)\right|}} \\
& \times \cos \left\{\frac{\hbar l A\left(E_{F}, k_{z}^{\mathrm{ext}}\right)}{e B}-2 \pi l \gamma+\frac{\pi}{4}\right\}+E_{\mathrm{total}}^{B=0}-\frac{\chi}{2} B^{2}
\end{aligned}
$$

with

$$
R_{D}=\exp \left(-2 \pi^{2} \frac{m_{c}\left(E_{F}, k_{z}^{\mathrm{ext}}\right) k_{B} T_{D}}{\hbar e B} l\right),
$$

where $A^{\prime \prime}\left(E_{F}, k_{z}^{\text {ext }}\right)$ and $\gamma$ and $g$ denote the curvature of the Fermi surface, $g$ factor, and phase correction, respectively. The factor $R_{D}$ is the so-called Dingle factor, and $T_{D}$ denotes the Dingle temperature, which is defined by $T_{D}=\hbar / 2 \pi k_{B} \tau$ [18]. In Eq. (7), $E_{\text {total }}^{B=0}$ and $-\chi B^{2} / 2$ denote the total energy

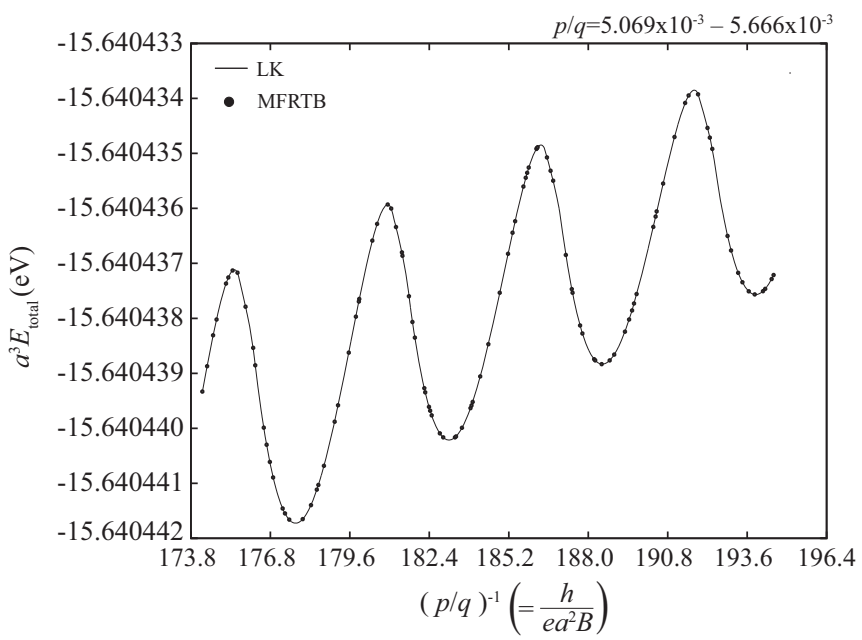

FIG. 8. Magnetic field dependences of the total energy (filled circle) in the $p / q$ range (c), $5.069 \times 10^{-3}-5.666 \times 10^{-3}$. The solid line and plots denote resultant fitted curves of the LK formula and calculation results of the MFRTB method, respectively. density for the zero magnetic field case and magnetization energy density, respectively, where $\chi$ is the susceptibility.

In order to analyze the amplitude of the dHvA oscillations, we determine values of $A^{\prime \prime}\left(E_{F}, k_{z}^{\text {ext }}\right), T_{D}, \gamma, A\left(E_{F}, k_{z}^{\text {ext }}\right), E_{\text {total }}^{B=0}$, and $\chi$ by fitting Eq. (7) to calculation results of the MFRTB method, where the value of $g$ is fixed at 2.0 because the MFRTB method is based on the Dirac equation. The method of least squares is employed in the fitting procedure. As the value of $m_{c}\left(E_{F}, k_{z}^{\text {ext }}\right)$, we use $m_{c}^{\mathrm{MFRTB}(\mathrm{C})}\left(E_{F}, k_{z}^{\text {ext }}\right)$ that is evaluated in the previous section [Figs. 7(a) and 7(b)]. Specifically, the following form is employed in the fitting procedure:

$$
\begin{gathered}
\frac{m_{c}^{\mathrm{MFRTB}(\mathrm{C})}\left(E_{F}, k_{z}^{\mathrm{ext}}\right)-m_{c}\left(E_{F}, k_{z}^{\mathrm{ext}}\right)}{m_{c}\left(E_{F}, k_{z}^{\mathrm{ext}}\right)} \\
=3.9467 \times 10^{-11} \times B^{2.7783} .
\end{gathered}
$$

This formula approximately represents both magnetic field dependences of $m_{c}^{\operatorname{MFRTB}(\mathrm{C})}\left(E_{F}, \pi / a\right)$ and $m_{c}^{\operatorname{MFRTB}(\mathrm{C})}\left(E_{F}, 0\right)$ that are shown in Figs. 7(a) and 7(b), respectively. Bearing in mind that both relations $A\left(E_{F}, 0\right)=A\left(E_{F}, \pi / a\right)$ and $A^{\prime \prime}\left(E_{F}, 0\right)=A^{\prime \prime}\left(E_{F}, \pi / a\right)$ hold for the case of the simple cubic lattice, there are six parameters that should be determined in the fitting procedure, i.e., $A\left(E_{F}, 0\right)\left[=A\left(E_{F}, \pi / a\right)\right]$, $T_{D}, A\left(E_{F}, 0\right)\left[=A\left(E_{F}, \pi / a\right)\right], \gamma, E_{\text {total }}^{B=0}$, and $\chi$. Values of $A^{\prime \prime}\left(E_{F}, k_{z}^{\text {ext }}\right)$ and $T_{D}$ are related to the oscillation amplitude in a different manner, and those of $A\left(E_{F}, k_{z}^{\mathrm{ext}}\right)$ and $\gamma$ determine the period and the shift of the oscillation, respectively. The nonoscillatory part of $E_{\text {total }}$ is determined by values of $E_{\text {total }}^{B=0}$ and $\chi$. Therefore, it is expected that we may readily determine

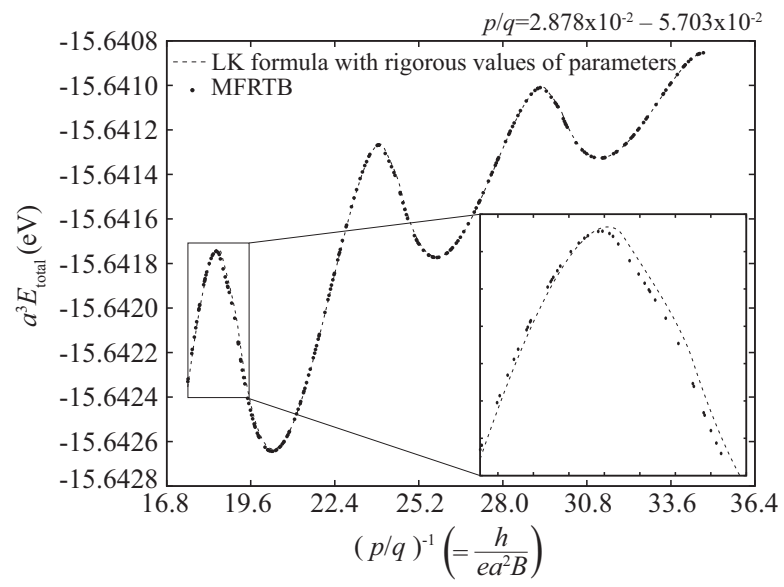

FIG. 9. Magnetic field dependence of the total energy for the cases of a magnetic field ranging from $p / q=2.878 \times 10^{-2}$ to $5.703 \times 10^{-2}$. The solid line and plots denote calculation results of the LK formula with rigorous values of parameters (Table II) and those of the MFRTB method, respectively. The inset is the magnified view of the dependence for the high magnetic field region. 
TABLE II. Rigorous values of parameters in the LK formula. Rigorous values are calculated by using the energy band structure for the zero magnetic field case.

\begin{tabular}{lcccc}
\hline \hline$A\left(E_{F}, k_{z}^{\text {ext }}\right)\left(\mathrm{m}^{-2}\right)$ & Period $\left(10^{-4} / \mathrm{T}\right)$ & $A^{\prime \prime}\left(E_{F}, k_{z}^{\text {ext }}\right)$ & $T_{\mathrm{D}}(\mathrm{K})$ & $m_{c}\left(E_{F}, k_{z}^{\text {ext }}\right) / m$ \\
\hline $2.47411 \times 10^{15}$ & 3.85826 & 8.6260636 & 0 & 0.10201 \\
\hline \hline
\end{tabular}

these values by fitting Eq. (7) to calculation results of the MFRTB. In the subsequent subsections, we discuss values of parameters that are related to the $\mathrm{dHvA}$ oscillations, i.e., $A\left(E_{F}, k_{z}^{\mathrm{ext}}\right), T_{D}, A^{\prime \prime}\left(E_{F}, k_{z}^{\mathrm{ext}}\right)$, and $\gamma$.

\section{B. Low $p / q$ region}

As mentioned in Sec. III C, the energy width of the cluster decreases with decreasing $p / q$. In addition, according to the discussion of Sec. $\mathrm{V}$, the deviation in the cyclotron effective mass is also negligible for the low $p / q$ region. Therefore, it is expected that the LK formula works well for the low $p / q$ region.

Values of $A^{\prime \prime}\left(E_{F}, k_{z}^{\text {ext }}\right), T_{D}, \gamma, A\left(E_{F}, k_{z}^{\text {ext }}\right), E_{\text {total }}^{B=0}$, and $\chi$ are determined individually for the following low $p / q$ regions: (a) $6.972 \times 10^{-4}-7.070 \times 10^{-4}$, (b) $3.079 \times 10^{-3}-$ $3.270 \times 10^{-3}$, (c) $5.069 \times 10^{-3}-5.666 \times 10^{-3}$. The resultant values are summarized in Table I. For reference, the resultant fitted curves (solid lines) and calculation results of the MFRTB method (plots) are shown in Fig. 8 for the case of the region (c). As shown in Fig. 8, the LK formula with resultant parameters (Table I) well reproduces the dHvA oscillations calculated by the MFRTB method. Oscillation periods obtained for the three regions are in good agreement with the rigorous value that is calculated from the energy band structure for the zero magnetic field case (see Table II). This agreement is consistent with the result of the previous paper [12]. Values of $T_{D}$ are nearly equal to zero, which means that a pseudo Dingle temperature does not appear in these $p / q$ regions. Values of $A^{\prime \prime}\left(E_{F}, k_{z}^{\mathrm{ext}}\right)$ are also in good agreement with the rigorous value that is given in Table II. Differences between the rigorous value of $A^{\prime \prime}\left(E_{F}, k_{z}^{\mathrm{ext}}\right)$ and fitted values are less than $0.01 \%$ for three $p / q$ regions.

The above-mentioned good agreements between resultant values of fitting parameters and rigorous values are consistent with the good agreement between $m_{c}\left(E_{F}, k_{z}^{\text {ext }}\right)$ and $m_{c}^{\mathrm{MFRTB}(\mathrm{X})}\left(E_{F}, k_{z}^{\text {ext }}\right)$ that is discussed in Sec. V. This suggests that the dHvA oscillations observed in these $p / q$ regions can be well described by the LK formula with good accuracy.

\section{High $p / q$ region}

Figure 9 shows the magnetic field dependence of the total energy for the case of $p / q$ ranging from $2.878 \times 10^{-2}$ to $5.703 \times 10^{-2}$. In Fig. 9, plots and dashed line denote calculation results of the MFRTB method and those of the LK formula with rigorous values of parameters that are given in Table II [25]. It is found from Fig. 9 that the total energies of the LK formula deviate from those of the MFRTB method with increasing $p / q$. The deviation observed in the high $p / q$ region implies that the $\mathrm{LK}$ formula does not work well in the high $p / q$ region.

Next, we discuss what kinds of errors will happen if we incorrectly apply the LK formula to the magnetic oscillation data for the high $p / q$ region. In a way similar to that in the previous subsection (Sec. VI B), we determine parameters of the LK formula by fitting Eq. (7) to calculation results of the MFRTB method. In the fitting procedure, the value of $E_{\text {total }}^{B=0}$ is fixed at the averaged value of results that are obtained for the low $p / q$ cases (Sec. VI B) [25]. We determine parameters of the LK formula by the following two fitting procedures:

(A) One is that $T_{D}, \gamma, A\left(E_{F}, k_{z}^{\text {ext }}\right)$, and $\chi$ are used as the fitting parameters while $A^{\prime \prime}\left(E_{F}, k_{z}^{\text {ext }}\right)$ is fixed at the rigorous value given in Table II.

(B) Another procedure is that $A^{\prime \prime}\left(E_{F}, k_{z}^{\text {ext }}\right), \gamma, A\left(E_{F}, k_{z}^{\text {ext }}\right)$, and $\chi$ are employed as the fitting parameters while $T_{D}$ is fixed at zero.

In the former procedure the deviation of the oscillation amplitude is attributed to that of $T_{D}$, while it is attributed to that of $A^{\prime \prime}\left(E_{F}, k_{z}^{\text {ext }}\right)$ in the latter procedure. These fitting procedures are done for five $p / q$ regions: (a) $6.309 \times 10^{-3}$ $8.559 \times 10^{-3}$, (b) $8.565 \times 10^{-3}-1.280 \times 10^{-2}$, (c) $1.2848 \times$ $10^{-2}-2.850 \times 10^{-2}$, (d) $2.878 \times 10^{-2}-5.703 \times 10^{-2}$, and (e) $5.727 \times 10^{-2}-2.487 \times 10^{-1}$.

Resultant values that are determined by the fitting procedures (A) and (B) are summarized in Tables III and IV, respectively. It is found from Table III that the pseudo Dingle temperature increases with $p / q$ and reaches a typical order of the Dingle temperature $[0.1-1(\mathrm{~K})]$ that is observed in experiments. The reason why the pseudo Dingle temperature increases with $p / q$ is that the energy width of the cluster increases with $p / q$ as mentioned in Sec. IV B. Thus, the reduction of the oscillation amplitude is caused by the energy width of the cluster non-negligibly even though $\tau$ is very large.

If the reduction of the oscillation amplitude is attributed to the value of $A^{\prime \prime}\left(E_{F}, k_{z}^{\text {ext }}\right)$ instead of the pseudo Dingle temperature, the resultant value of $A^{\prime \prime}\left(E_{F}, k_{z}^{\text {ext }}\right)$ gradually increases with $p / q$ (Table IV). This would cause the overestimation

TABLE III. Values of parameters for high $p / q$ regions. These values are determined by the fitting procedure (A).

\begin{tabular}{|c|c|c|c|c|}
\hline Range of $p / q$ & Period $\left(10^{-4} / \mathrm{T}\right)$ & $\gamma$ & $A^{\prime \prime}\left(E_{F}, k_{z}^{\mathrm{ext}}\right)$ & $T_{\mathrm{D}}(\mathrm{K})$ \\
\hline $6.309 \times 10^{-3}-8.559 \times 10^{-3}$ & 3.85845 & 0.498 & 8.6260636 & $1.22 \times 10^{-4}$ \\
\hline $8.565 \times 10^{-3}-1.280 \times 10^{-2}$ & 3.85870 & 0.496 & 8.6260636 & $1.48 \times 10^{-2}$ \\
\hline $1.2848 \times 10^{-2}-2.850 \times 10^{-2}$ & 3.86005 & 0.492 & 8.6260636 & $1.32 \times 10^{-1}$ \\
\hline $2.878 \times 10^{-2}-5.703 \times 10^{-2}$ & 3.86642 & 0.483 & 8.6260636 & $1.05 \times 10^{0}$ \\
\hline $5.727 \times 10^{-2}-2.487 \times 10^{-1}$ & 3.88140 & 0.469 & 8.6260636 & $3.66 \times 10^{1}$ \\
\hline
\end{tabular}


TABLE IV. Values of parameters for high $p / q$ regions. These values are determined by the fitting procedure (B).

\begin{tabular}{lccc}
\hline \hline Range of $p / q$ & Period $\left(10^{-4} / \mathrm{T}\right)$ & $\gamma$ & $A^{\prime \prime}\left(E_{F}, k_{z}^{\text {ext }}\right)$ \\
\hline $6.309 \times 10^{-3}-8.559 \times 10^{-3}$ & 3.85844 & 0.498 & 8.6263427 \\
$8.565 \times 10^{-3}-1.280 \times 10^{-2}$ & 3.85870 & 0.496 & 8.6287488 \\
$1.2848 \times 10^{-2}-2.850 \times 10^{-2}$ & 3.86004 & 0.492 & 8.6397337 \\
$2.878 \times 10^{-2}-5.703 \times 10^{-2}$ & 3.86655 & 0.483 & 8.6724848 \\
$5.727 \times 10^{-2}-2.487 \times 10^{-1}$ & 3.88505 & 0.467 & 9.0602895 \\
\hline \hline
\end{tabular}

of the curvature of the Fermi surface $A^{\prime \prime}\left(E_{F}, k_{z}^{\text {ext }}\right)$ if the LK formula was incorrectly utilized in analyzing the oscillation amplitude for the high $p / q$ region.

In both Tables III and IV, oscillation periods gradually increase with $p / q$, so that the difference between the oscillation period and the rigorous one increases with $p / q$. This $p / q$ dependence of the period is consistent with the result of the previous paper [12]. The difference in the period implies that $A\left(E_{F}, k_{z}^{\text {ext }}\right)$ would be underestimated if the LK formula was incorrectly applied to the magnetic oscillation data for the high $p / q$ region. Although the value of $\gamma$ is close to that for the free electron case $(\gamma=0.5)$ in the low $p / q$ regions (Table I), it gradually deviates from 0.5 with increasing $p / q$ (Tables III and IV). This means that the free electron model becomes unsuitable for the system immersed in the high magnetic field with high $p / q$.

It should be mentioned that the above-mentioned reduction of the oscillation amplitude may be observed experimentally depending on the system. As mentioned in Sec. IV B, the energy width of the cluster depends on $p / q[11,20]$. In the case of the simple cubic lattice, it is found from Table III or IV that the pseudo Dingle temperature or overestimation of $A^{\prime \prime}\left(E_{F}, k_{z}^{\text {ext }}\right)$ becomes non-negligible when $p / q$ is more than $2.878 \times 10^{-2}$ [regions (d) and (e)]. The rational number $p / q \approx 2.878 \times 10^{-2}$ corresponds to $400 \mathrm{~T}$ for the system with $a=0.543 \mathrm{~nm}$. If we consider the system with a period that is one order of magnitude longer than $a$, then $p / q \approx 2.878 \times$ $10^{-2}$ corresponds to $B \approx 4 \mathrm{~T}$, which is an experimentally available magnetic field. For example, since graphene placed on a hBN substrate has a long period (about $15 \mathrm{~nm}$ ) of the moiré superlattice potential [21-24] as mentioned in Sec. IV C, there is a possibility that the pseudo Dingle temperature and/or the overestimation of $A^{\prime \prime}\left(E_{F}, k_{z}^{\text {ext }}\right)$ are observable experimentally in a system with a long period, such as a superlattice system.

\section{CONCLUDING REMARKS}

The MFRTB method is a first-principles calculation method for electronic structures of metals immersed in a magnetic field. On the basis of electronic structures calculated by the MFRTB method, we investigate magnetic properties of a simple cubic lattice system with $s$ electrons that is immersed in a uniform magnetic field. The electronic structure calculated by the MFRTB method has the following property that becomes the key point for describing the magnetic oscillations of metals:

(1) The electronic structure calculated by the MFRTB method has a fine energy-level structure: The cluster of energy bands that lie within a small energy width corresponds to the semiclassical energy level.
With the aid of this knowledge, we obtain the description for the conventional dHvA oscillations:

(2) Every time the cluster of energy bands that corresponds to the semiclassical energy level crosses the Fermi energy, the slope of the total energy with respect to the magnetic field is changed, which causes the periodic change of the magnetization.

The fine energy-level structure that is found by the MFRTB method causes the following phenomena:

(3) When energy bands that are constituents of the cluster cross the Fermi energy, additional oscillation peaks of the magnetization emerge together with the conventional $\mathrm{dHvA}$ oscillations.

(4) Due to the energy width of the cluster, an unexpected reduction of the oscillation amplitude occurs. This reduction causes the pseudo Dingle temperature and/or the overestimation of the curvature of the Fermi surface.

These phenomena cannot be explained by the semiclassical approach and are found by means of the MFRTB method. We also discuss the observability of phenomena (3) and (4), and we achieve the following result:

(5) There is a possibility that the above-mentioned phenomena (3) and (4) are observable in experiments. For example, phenomena (3) and (4) may be observed in some systems with a long period, such as a superlattice system [21-24].

Thus, beyond the semiclassical approach of the Bloch electron immersed in a magnetic field, the MFRTB method provides a first-principles way to describe physical phenomena observed in a magnetic field. Especially, the MFRTB method can predict physical phenomena [such as (3) and (4)] that cannot be described by the semiclassical approach. This knowledge would provide one of the possible scenarios of magnetic oscillations.

Finally, we comment on the future lines of the discussion about the effect of finite temperature on phenomena (3) and (4). When the effect of finite temperatures is discussed, DOSs that have already been obtained in the present work can be utilized in calculating the free energy and magnetization. Then, we may investigate the temperature dependence of the intensity of additional oscillation peaks. In addition, by estimating the pseudo Dingle temperature from the temperature and magnetic field dependences of the magnetization, we may discuss the effect of finite temperatures on the unexpected reduction of the oscillation amplitude.

\section{ACKNOWLEDGMENT}

This work was partially supported by JSPS KAKENHI Grants No. JP26400354, No. JP26400397, and No. JP16H00916. 
[1] W. J. de Haas and P. M. van Alphen, Proc. Netherland R. Acad. Sci. 33, 680 (1930).

[2] W. J. de Haas and P. M. van Alphen, Proc. Netherland R. Acad. Sci. 33, 1106 (1930).

[3] W. J. de Haas and P. M. van Alphen, Proc. Netherland R. Acad. Sci. 35, 454 (1932).

[4] L. Onsager, Philos. Mag. 43, 1006 (1952).

[5] D. Shoenberg, Magnetic Oscillation in Metals (Cambridge University Press, Cambridge, 1984).

[6] M. R. Norman and D. D. Koelling, in Handbook on the Physics and Chemistry of Rare Earths, edited by K. A. Gschneidner, Jr., L. Eyring, G. H. Lander, and G. R. Choppin (North-Holland, Amsterdam, 1993), Vol. 17, Chap. 110.

[7] Y. Onuki and A. Hasegawa, in Handbook on the Physics and Chemistry of Rare Earths, edited by K. A. Gschneidner, Jr. and L. Eyring (North-Holland, Amsterdam, 1995), Vol. 20, Chap. 135.

[8] N. W. Ashcroft and N. D. Mermin, Solid State Physics (W. B. Saunders, Philadelphia, 1976), Chap. 14.

[9] J. Kübler, Theory of Itinerant Electron Magnetism (Oxford University Press, Oxford, 2000), Chap. 1.

[10] I. M. Lifshitz and A. M. Kosevich, Sov. Phys. JETP 2, 636 (1956).

[11] K. Higuchi, D. B. Hamal, and M. Higuchi, Phys. Rev. B 91, 075122 (2015).

[12] D. B. Hamal, M. Higuchi, and K. Higuchi, Phys. Rev. B 91, 245101 (2015).

[13] T. Goto, H. Yamada-Kaneta, Y. Saito, Y. Nemoto, K. Sato, K. Kakimoto, and S. Nakamura, J. Phys. Soc. Jpn. 75, 044602 (2006).

[14] S. Baba, T. Goto, Y. Nagai, M. Akatsu, H. Watanabe, K. Mitsumoto, T. Ogawa, Y. Nemoto, and H. Yamada-Kaneta, J. Phys. Soc. Jpn. 80, 094601 (2011).

[15] K. Okabe, M. Akatsu, S. Baba, K. Mitsumoto, Y. Nemoto, H. Yamada-Kaneta, T. Goto, H. Saito, K. Kashima, and Y. Saito, J. Phys. Soc. Jpn. 82, 124604 (2013).
[16] K. Mitsumoto, M. Akatsu, S. Baba, R. Takasu, Y. Nemoto, T. Goto, H. Yamada-Kaneta, Y. Furumura, H. Saito, K. Kashima, and Y. Saito, J. Phys. Soc. Jpn. 83, 034702 (2014).

[17] D. R. Hofstadter, Phys. Rev. B 14, 2239 (1976).

[18] R. B. Dingle, Proc. R. Soc. London A 211, 517 (1952).

[19] We refer the set of energy bands as a "cluster". This is consistent with the previous definition of a cluster [11], which is mentioned in Sec. IV B.

[20] Since the number of allowed bands is proportional to $q^{\prime}$, the energy width of the allowed band is inversely proportional to $q^{\prime}$ in the case of $B \propto 1 / q^{\prime}$. In the case of $B \propto p / q$, the allowed band in the case of $B \propto 1 / q^{\prime}$ splits into multiple allowed bands that form the cluster. Therefore, the energy width of the cluster in the case of $B \propto p / q$ is roughly equal to the energy width of the allowed band in the case of $B \propto 1 / q^{\prime}$. Therefore, the energy width of the cluster is inversely proportional to $q^{\prime} \approx q / p$.

[21] L. A. Ponomarenko, R. V. Gorbachev, G. L. Yu, D. C. Elias, R. Jalil, A. A. Patel, A. Mishchenko, A. S. Mayorov, C. R. Woods, J. R. Wallbank, M. Mucha-Kruczynski, B. A. Piot, M. Potemski, I. V. Grigorieva, K. S. Novoselov, F. Guinea, V. I. Falko, and A. K. Geim, Nature (London) 497, 594 (2013).

[22] C. Dean, L. Wang, P. Maher, C. Forsythe, F. Ghahari, Y. Gao, J. Katoch, M. Ishigami, P. Moon, M. Koshino, T. Taniguchi, K. Watanabe, K. Shepard, J. Hone, and P. Kim, Nature (London) 497, 598 (2013).

[23] B. Hunt, J. Sanchez-Yamagishi, A. Young, M. Yankowitz, B. LeRoy, K. Watanabe, T. Taniguchi, P. Moon, M. Koshino, P. Jarillo-Herrero, and R. Ashoori, Science 340, 1427 (2013).

[24] G. L. Yu, R. V. Gorbachev, J. S. Tu, A. V. Kretinin, Y. Cao, R. Jalil, F. Withers, L. A. Ponomarenko, B. A. Piot, M. Potemski, D. C. Elias, X. Chen, K. Watanabe, T. Taniguchi, I. V. Grigorieva, K. S. Novoselov, V. I. Falko, A. K. Geim, and A. Mishchenko, Nat. Phys. 10, 525 (2014).

[25] We use $-15.6404163224 \mathrm{eV}, 9.3407 \times 10^{-6}$, and 0.498 as the values of $E_{\text {total }}^{B=0}, \mu_{0} \chi$, and $\gamma$, respectively, which are obtained by averaging resultant values for three low $p / q$ regions (Sec. VIB). 